\title{
Nutrient Budget for Optimal Oil Palm (Elaeis guineensis Jacq) Yield on Coastal Plain Sands Soils of Akwa Ibom State Nigeria
}

\section{Jude Chukwuma Obi* , Bassey Thomas Udoh}

Department of Soil Science, University of Uyo, Uyo, Nigeria.

Email: *obijbc@yahoo.com

Received May $11^{\text {th }}, 2012$; revised June $15^{\text {th }}, 2012$; accepted June $30^{\text {th }}, 2012$

\begin{abstract}
The objective of the study was to establish approximate relationships between yield and soil nutrients in oil palm production. The study was conducted in Nigerian Institute for Oil Palm Research (NIFOR) substation Ibesit ekoi in Oruk Anam Local Government Area of Akwa Ibom State Nigeria. Soil, rainfall and yield data were collected from oil palm plantation established 49, 29, 9 and 0 (control) years ago in an area underlain by coastal plain sands. Descriptive statistics, analysis of variance and multiple stepwise regression analysis were used to study variations, effect of land use on soil properties at different depths and contributions of various soil nutrients at different depths to the yield (fresh fruit bunch 'FFB' and palm oil) of oil palm. Results of coefficient of variability revealed that approx. $45.5 \%$ of the variables were highly variable including available phosphorus, extractable zinc, FFB and palm oil, while others were either least or moderately variable. Oil palm trees influenced soil development with its effect on silt content at $30-60 \mathrm{~cm}$ depth. Uptake of phosphorus in oil palm land use system decreases with depth. This was further confirmed by the relative contribution of available phosphorus to FFB yield that decreased from the surface of the soil downwards. Extractable zinc contents of oil palm land use were not significantly different from each other (ranging between $9.65 \mathrm{mg} \cdot \mathrm{kg}^{-1}$ and 7.84 $\left.\mathrm{mg} \cdot \mathrm{kg}^{-1}\right)$ but significantly different from the control $\left(23.99 \mathrm{mg} \cdot \mathrm{kg}^{-1}\right)$. In the modeling process, it was observed that the absolute contribution of texture was minimal while exchangeable sodium was highest (i.e. 66.5\%) in the quantity of oil palm production. Also extractable copper and zinc were found to have made large contributions to FFB and oil palm. Oil palm (Elaeis guineensis) is a high-yielding source of edible and technical oils but requires proper knowledge and precise administration of nutrient demands for management of a major production constraint which is soil fertility.
\end{abstract}

Keywords: Soil Nutrient Budget; Oil Palm; Micronutrients; Modeling; Soil Development

\section{Introduction}

It is generally agreed that the Oil palm (Elaeis guineensis) originated in the tropical rain forest region of West Africa. It posses high economic importance because it is a high-yielding source of edible and technical oils. Oil palm is now grown as a plantation crop in most countries with high rainfall (minimum $1600 \mathrm{~mm} / \mathrm{yr}$ ) in tropical climates within $10^{\circ}$ of the equator. Hence, the oil palm (Elaeis guineensis) is an important economic tree crops in the tropics. The African oil palm, Elaeis guineensis Jacq, is a member of the Arecaceae family along with coconut and date palms. Oil palm is the world's number one fruit crop, according to Rieger [1], world production is approximately $153,578,600$ million ton, which is approximately twice any other fruit crop production. Oil palm is produced in 42 countries worldwide on about 27

*Corresponding author. million acres. Average yields are 10,000 lbs/acre (i.e. $1126.761 \mathrm{~kg} \cdot \mathrm{ha}^{-1}$ ), and per acre yield of oil from African oil palm is more than 4 -folds that of any other oil crop.

The most important constraint to oil palm production is soil fertility. It was estimated that more than 95 percent of oil palms grown in Southeast Asia are on acid, low fertility and highly weathered soils [2]. This was corroborated in the study conducted by Imogie et al. [3] in Bayelsa State of Nigeria. Soyebo, et al. [4] further observed that approximately 93 percent of oil palms found in Osun State (Nigeria) are in wild comprising mostly degraded lands. Soil physical properties such as depth, texture and structure are important factors in determining suitability for large scale oil palm production [2]. These are based on the required clay loam texture that imposes friable consistency, capacity to permits extensive root development, firm anchorage, and capacity to stores sufficient water and plant nutrients. Majority of oil palm 
roots are found within the first $60 \mathrm{~cm}$ of the soil [2]. Therefore, these requisite soil conditions and nutrient status that favour growth and development is indispensible within the $0-60 \mathrm{~cm}$ depth, yet the importance of firm anchorage creates the need for deeper soils (greater than $90 \mathrm{~cm}$ ).

Large quantity of dry matter is generated in oil palm production. These comprised those in the fresh fruit bunch and large quantities sequestered in the standing biomass. Therefore, oil palm has an established high demand for nutrients. These nutrients must be supplied as amendments and in a suitable balance [5] for yields to be maximized and environment sustained as they may not be released on sustained basis in the soil. Ng [6] suggested that nutrient budget of oil palm must be compared with the soils capacity while designing fertilizer or nutrient management scheme for economic production. For instance, increased supply of nitrogen and potassium without an adequate supply of magnesium on soils with a low magnesium status can lead to the development of Orange Frond symptoms in younger palms (a nutritional disorder which later depresses growth and eventually yields). Appropriate micronutrients in the nutrient budget will equally enhance the efficiency of use of nitrogen $(\mathrm{N})$, phosphorus $(\mathrm{P})$ and potassium $(\mathrm{K})$ and also meet the crop's needs. The deficiency of micronutrients is the nutritional disorders that manifest with common incidence of chlorotic and desiccated leaves due copper $(\mathrm{Cu})$ and zinc $(\mathrm{Zn})$ deficiency $[7,8]$.

The objective of this study was to establish approximate relationships between yield and soil nutrients in oil palm production.

\section{Materials and Methods}

\subsection{Site Description}

The study was conducted in Nigerian Institute for Oil Palm Research (NIFOR) substation Ibesit ekoi in Oruk Anam Local Government Area of Akwa Ibom State Nigeria. Oruk Anam Local Government Area is bounded within latitudes $4^{\circ} 45^{\prime}$ and $5^{\circ} 00^{\prime} \mathrm{N}$ and longitudes $7^{\circ} 30^{\prime}$ and $7^{\circ} 45^{\prime} \mathrm{E}$. The climate is humid tropical characterised by distinct rainy (February/March-November) and dry (November-February/March) season. Rainfall ranges from $3000 \mathrm{~mm}$ along the Atlantic coast to $2000 \mathrm{~mm}$ in the hinterland [9]. The mean daily temperature is about $29^{\circ} \mathrm{C}$, relative humidity approximately $80 \%$ and sunshine approximately 5 hours per day but changes during the year in response to changes in climate and possesses. The overall topography is typical of unconsolidated marine and fluvial deposit formation. The State falls within the sedimentary areas of Nigeria with up to $80 \%$ of the soil formed on coastal plain sands $(>70 \%)$ and alluvium [9-
11]. Oruk Anam Local Government Area falls within the area covered by coastal plain sands. Soils on coastal plain sands are normally deep, dominantly sandy with low clay, organic matter content and $\mathrm{pH}$ [12]. The soils are well drained, deeply weathered and formed on sandy, coarse-loamy materials, have udic moisture regime, isohypertermic temperature regime and broadly classified as ultisol.

\subsection{Field Work and Laboratory Analysis}

The study was carried out on oil palm plantation in NIFOR substation in 2010. The sampling scheme was designed based on various land uses characterized by ages of oil palm trees. These include oil palm trees established between 1960 and 2000 and divided into three groups separated at twenty years intervals. Namely 49 years, 29 years, 9 years and 0 year (control) which resided at the accompanying fallow land. Mean yield data (Fresh Fruit Bunch weight) and quantity of oil (palm oil) produced therein (in $\mathrm{kg} \cdot \mathrm{ha}^{-1} \cdot \mathrm{yr}^{-1}$ and litres $\cdot \mathrm{ha}^{-1} \cdot \mathrm{yr}^{-1}$ respectively) for each of the three groups was collected. In each land use, four replicate sample plots were demarcated (corresponding to the upper, middle, lower and valley bottom slope positions) and 10 composite soil samples were randomly collected from $0-60 \mathrm{~cm}$ depth comprising 0 $15 \mathrm{~cm}, 15-30 \mathrm{~cm}$ and $30-60 \mathrm{~cm}$ depths. The available annual (1977 to 2010) rainfall data was collected from the Uyo (the nearest) weather station which is approximately $25 \mathrm{~km}$ (as the crow flies) away from NIFOR substation. Annual yield data (both for weight of fresh fruit bunch (FFB) and the corresponding quantity of oil produced were collected for each of the oil palm trees age groups (land uses).

The soil samples were air dried, pulverized and made to pass through 2-mm mesh sieve. Particle size distribution was carried out through hydrometer method [13]. Organic carbon was determined by dichromate oxidation [14] method. Soil pH was determined in a 1:2.5 (soil: water) solution using $\mathrm{pH}$ meter [15]. Exchangeable bases, available phosphorus (avail P) and micronutrients were extracted with Mehlick No. 3 extraction [16]. Potassium $(\mathrm{K})$ and sodium $(\mathrm{Na})$ content were read with the aid of flame emission spectroscopy. Calcium $\left(\mathrm{Ca}^{2+}\right)$ and magnesium $\left(\mathrm{Mg}^{2+}\right)$ and micronutrients (iron, zinc, copper and manganese) were read with the aid of atomic absorption spectroscopy (AAS), while total phosphorus was determined colorimetrically. Exchangeable acidity was extracted with un-buffered potassium chloride solution and titration with $0.01 \mathrm{M}$-solution of sodium hydroxide to the first permanent pink endpoint as described by Anderson and Ingram [17]. Effective cation exchange capacity (ECEC) was determined through summation [18]. 


\subsection{Statistical Analysis}

Data was collected in Randomized Complete Block Design, therefore analysis of variance (ANOVA) was used to study the effect of oil palm (i.e. land uses) on the soil properties, while significantly different means were separated using least significant difference (LSD) at 5\% probability level. Statistics of dispersion, normality of distribution [19] and measure of central tendencies were carried out. Multiple stepwise regression analyses were carried out with either fresh fruit bunch (FFB) or quantity of palm oil as the dependent variable to model their relationship with soil nutrients as independent variables (either micronutrient with particle size fraction (PSF) or others with PSF in turns. All statistical analyses were carried out using SAS [20].

\section{Results and Discussion}

The descriptive statistics of the data generated from the study were shown in Table 1. This comprise mean, median and mode that measures location of central tendency, skewness and kurtosis was used to estimate normality of distribution while standard error and coefficient of variation were used as estimates of variability. The mean median and mode of the variable measured were similar. Considering the levels of significances observed in univariate normal distribution shown in Table 1, some variables were either skewed or kurtous $(\operatorname{Pr}<\mathrm{W})$ which was based on Shipiro Wilk [19]. Even the non-normally distributed variables were not dominated by outliers and therefore could be assumed to have originated from same population. According to the Wilding [21] classification, variables that had coefficient of variation $(\mathrm{CV})$ greater than $35 \%$ (i.e. neither least nor moderately, but highly variable) included silt, (44.9\%), organic carbon (57.6\%), available phosphorus $(48.7 \%)$, electrical conductivity (56\%), exchangeable calcium (41.3\%), exchangeable acidity (39.0\%), manganese (54.2\%) and zinc (65.6\%). In addition are the Fresh fruit bunch and oil palm that had coefficient of variation of $49.6 \%$ and $55.6 \%$ respectively. Other variables including rainfall were either least variable $(<15 \%)$ or moderately variable $(>15 \%<35 \%)$. High variability of available phosphorus and other properties had been reported as common occurrence in soils especially due to intrinsic variation, land use and management $[22,23]$. But the high CV of extractable zinc could be of concern due to its important role in growth and development of oil palm.

The properties of soil at various locations as influenced by age of oil palm were compared. The particle size fractions were dominated with sand fractions (coarse and fine sand) with overall mean of approximately $>836$ $\mathrm{g} \cdot \mathrm{kg}^{-1}$ (Table 1). Coastal plain sands soils are character- ised by dominance of sand fraction with coarse sand contributing more than fine sand [12]. There were generally similarities in the distribution of the particle size fractions (Table 2) and ratios of exchangeable bases (Table 4) in the various land uses (i.e. ages of oil palm including the control) at all depths with the exception of silt content at the $60 \mathrm{~cm}$ depth. These were confirmations that the soils of the study area are typically coastal plain sands soils. The significant differences of silt content at the $60 \mathrm{~cm}$ depth could be attributed to the effect of land use on soil development [24] as this is the effective depth for oil palm nutrition beyond which anchorage becomes the important factor. Additionally it has been reported that trees deepen soils, increases weathering and soil development through their penetration and focusing moisture flux, producing organic acids, facilitating microbial activity and displacing loosened clasts [25].

The soil properties that vary significantly $(\mathrm{p}<0.05)$ among those indicated in Table 3 (i.e. excluding particle size fraction (Table 2), ratios of exchangeable bases (Table 4) and micronutrients (Table 5)) include available phosphorus at $15 \mathrm{~cm}$ and $30 \mathrm{~cm}$ depths, base saturation at $30 \mathrm{~cm}$ and $60 \mathrm{~cm}$ depths, exchangeable sodium at $15 \mathrm{~cm}, 30 \mathrm{~cm}$ and $60 \mathrm{~cm}$ depths and exchangeable acidity at $15 \mathrm{~cm}$ and $60 \mathrm{~cm}$ depths. The result in Table 3 indicated that oil palm significantly influenced the available phosphorus in the soil especially at $0-15 \mathrm{~cm}$ depth which had the control containing higher amount of available phosphorus. This effect repeated at $15-30 \mathrm{~cm}$ depth, but with location (i.e. land use) that experience longer period of oil palm production (49 years) possessing significantly lower amount of available phosphorus than the control. This effect decreases with depth as significant difference was not observed at $30-60 \mathrm{~cm}$ depth. Hence it could be inferred that utilization of phosphorus in oil palm land use system decreases with depth. This was further confirmed by the relative contribution of available phosphorus to FFB that decreased from the surface of the soil downwards and inverted in the corresponding quantity of palm oil which decreased downwards (Table 7). Suggesting that the amount of available phosphorus may need to be critically adjusted in the nutrient management scheme for acceptable balance between yield in FFB and corresponding palm oil produced therein. In contrast to the available phosphorus, base saturation at the control (79.1\%) was significantly lower than that at the oldest plantation $(86.6 \%)$ at the $15-30$ $\mathrm{cm}$ depth. But more typical is the scenario at the $30-60$ $\mathrm{cm}$ depth that base saturation decreased from 49 years to 0 years (control). The ratios of the exchangeable bases were not significantly different which still confirmed that the soils were actually from similar parent material [26-28] and that oil palm production or uptake has not 
Table 1. Descriptive statistics of the soil properties in the palm plantation.

\begin{tabular}{|c|c|c|c|c|c|c|c|c|c|}
\hline \multicolumn{2}{|l|}{ 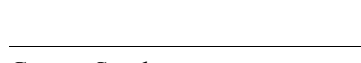 } & Mean & Median & Mode & SE & $\mathrm{CV}$ & Skew & Kurtosis & $\operatorname{Pr}<\mathrm{W}$ \\
\hline \multicolumn{2}{|l|}{ Coarse Sand } & 454.9 & 444.0 & 444.0 & 1.31 & 20.01 & -0.09 & -0.71 & $0.578^{*}$ \\
\hline \multicolumn{2}{|l|}{ Fine Sand } & 381.7 & 380.0 & 320.0 & 1.21 & 22.02 & 0.19 & -0.79 & $0.24^{*}$ \\
\hline \multicolumn{2}{|l|}{ Silt } & 47.6 & 41.0 & 41.0 & 0.31 & 44.9 & 0.80 & 2.17 & 0.00 \\
\hline \multicolumn{2}{|l|}{ Clay } & 115.4 & 115.0 & 155.0 & 0.56 & 33.74 & 0.31 & -0.63 & $0.01^{*}$ \\
\hline \multicolumn{2}{|l|}{$\mathrm{pH}\left(\mathrm{H}_{2} \mathrm{O}\right)$} & 5.77 & 5.76 & 5.76 & 0.02 & 2.46 & 1.02 & 1.84 & 0.00 \\
\hline \multicolumn{2}{|c|}{ Organic Carbon } & 1.05 & 0.95 & 1.40 & 0.89 & 57.6 & 0.18 & -0.83 & $0.97^{*}$ \\
\hline \multicolumn{2}{|c|}{ Available Phosphorus } & 7.33 & 6.60 & 6.60 & 0.51 & 48.46 & 0.66 & -0.28 & $0.03^{*}$ \\
\hline \multicolumn{2}{|c|}{ Base Saturation } & 81.34 & 81.50 & 81.10 & 0.97 & 8.23 & -0.31 & 1.16 & $0.33^{*}$ \\
\hline \multicolumn{2}{|c|}{ Electrical Conductivity } & 0.01 & 0.01 & 0.01 & 0.001 & 55.95 & 2.97 & 10.99 & 0.00 \\
\hline \multirow{5}{*}{ Exchangeable } & Calcium & 3.49 & 3.20 & 2.67 & 0.21 & 41.34 & 2.06 & 5.41 & 0.00 \\
\hline & Magnesium & 5.59 & 5.33 & 5.86 & 0.22 & 27.57 & 1.81 & 6.28 & 0.00 \\
\hline & Potassium & 0.06 & 0.06 & 0.05 & 0.00 & 27.24 & -0.84 & 2.23 & 0.00 \\
\hline & Sodium & 0.05 & 0.05 & 0.04 & 0.00 & 14.11 & 0.66 & -0.51 & 0.00 \\
\hline & Acidity & 2.05 & 1.76 & 1.60 & 0.12 & 39.04 & 1.37 & 2.85 & 0.00 \\
\hline \multicolumn{2}{|l|}{ ECEC } & 11.22 & 10.75 & - & 0.45 & 27.64 & 1.55 & 3.39 & 0.00 \\
\hline \multirow{4}{*}{ Extractable } & Manganese & 9.88 & 9.21 & 6.01 & 0.77 & 54.2 & 0.71 & 0.12 & $0.08^{*}$ \\
\hline & Zinc & 11.51 & 8.64 & - & 1.09 & 65.6 & 1.983 & 4.01 & 0.00 \\
\hline & Copper & 11.69 & 10.72 & - & 0.55 & 32.57 & 0.75 & 0.35 & $0.04^{*}$ \\
\hline & Iron & 100.96 & 100.51 & 78.78 & 2.06 & 14.13 & 0.27 & -0.31 & $0.28^{*}$ \\
\hline \multicolumn{2}{|c|}{ Fresh Fruit Bunch } & 333.67 & 296.00 & 261.00 & 47.83 & 49.63 & 0.39 & -1.65 & 0.00 \\
\hline \multicolumn{2}{|c|}{ Palm oil (litres) } & 2207.01 & 1827.34 & 1995.70 & 354.31 & 55.61 & 0.52 & -1.65 & 0.00 \\
\hline \multicolumn{2}{|l|}{ Rainfall } & 7861.13 & 7828.34 & 7997.73 & 186.57 & 9.49 & 0.18 & -0.17 & $0.92^{*}$ \\
\hline
\end{tabular}

${ }^{*}$ Neither significant at $1 \%$ nor $5 \%$; SE: standard error; $\mathrm{CV}$ : coefficient of variation; Skew: skewness.

Table 2. Particle size fractions $\left(\mathrm{g} \cdot \mathrm{kg}^{-1}\right)$ of soil of the palm plantation.

\begin{tabular}{|c|c|c|c|c|c|}
\hline & \multicolumn{4}{|c|}{ Age of Oil Palm Plantation (years) } & \multirow[b]{2}{*}{$\mathrm{LSD}_{0.05}$} \\
\hline & 0 & 9 & 29 & 49 & \\
\hline \multicolumn{6}{|c|}{0 - 15 cm Depth } \\
\hline Coarse Sand & 467.5 & 432.5 & 467.5 & 412.5 & 142.8 \\
\hline Fine Sand & 335.0 & 430.0 & 395.0 & 395.0 & 116.0 \\
\hline Silt & 66.8 & 46.0 & 39.5 & 56.8 & 50.8 \\
\hline Clay & 100.8 & 91.0 & 98.0 & 135.8 & 62.5 \\
\hline \multicolumn{6}{|c|}{15 - 30 cm Depth } \\
\hline Coarse Sand & 504.0 & 389.0 & 402.5 & 299.3 & 326.8 \\
\hline Fine Sand & 325.0 & 350.0 & 425.0 & 430.0 & 130.7 \\
\hline Silt & 59.5 & 35.0 & 51.8 & 40.3 & 28.1 \\
\hline Clay & 111.5 & 105.0 & 120.8 & 90.8 & 58.4 \\
\hline \multicolumn{6}{|c|}{30 - 60 cm Depth } \\
\hline Coarse Sand & 472.5 & 365.3 & 391.0 & 311.0 & 300.2 \\
\hline Fine Sand & 360.0 & 486.0 & 395.0 & 541.0 & 347.0 \\
\hline Silt & 44.5 & 60.3 & 37.5 & 76.0 & 38.3 \\
\hline Clay & 123.0 & 183.5 & 176.5 & 147.0 & 127.7 \\
\hline
\end{tabular}


Table 3. Some chemical properties of soil of the palm plantation.

\begin{tabular}{|c|c|c|c|c|c|c|}
\hline & & \multicolumn{4}{|c|}{ Age of Oil Palm Plantation (Years) } & \multirow[b]{2}{*}{$\mathrm{LSD}_{0.05}$} \\
\hline & & 0 & 9 & 29 & 49 & \\
\hline \multicolumn{7}{|c|}{0 - 15 cm Depth } \\
\hline \multicolumn{2}{|l|}{$\mathrm{pH}\left(\mathrm{H}_{2} \mathrm{O}\right)$} & 5.89 & 5.74 & 5.89 & 5.80 & 0.29 \\
\hline \multicolumn{2}{|c|}{ Organic Carbon } & 1.43 & 1.33 & 0.85 & 1.43 & 0.70 \\
\hline \multicolumn{2}{|c|}{ Available Phosphorus } & 7.35 & 3.87 & 3.85 & 3.20 & 2.73 \\
\hline \multicolumn{2}{|l|}{ Base Saturation } & 81.78 & 75.75 & 83.65 & 82.95 & 7.75 \\
\hline \multicolumn{2}{|c|}{ Electrical Conductivity } & 0.010 & 0.015 & 0.020 & 0.0125 & 0.02 \\
\hline \multirow{5}{*}{ Exchangeable } & Calcium & 5.60 & 5.19 & 5.20 & 5.46 & 2.38 \\
\hline & Magnesium & 3.58 & 3.34 & 3.07 & 3.87 & 2.93 \\
\hline & Potassium & 0.075 & 0.055 & 0.070 & 0.057 & 0.03 \\
\hline & Sodium & 0.05 & 0.04 & 0.04 & 0.05 & 0.006 \\
\hline & Acidity & 2.00 & 2.77 & 1.60 & 1.72 & 1.13 \\
\hline \multicolumn{2}{|c|}{ Effective Cation Exchange Capacity } & 11.19 & 11.38 & 9.97 & 11.16 & 5.82 \\
\hline \multicolumn{7}{|c|}{15 - 30 cm Depth } \\
\hline \multicolumn{2}{|l|}{$\mathrm{pH}\left(\mathrm{H}_{2} \mathrm{O}\right)$} & 5.78 & 5.70 & 5.88 & 5.72 & 0.23 \\
\hline \multicolumn{2}{|c|}{ Organic Carbon } & 1.10 & 1.10 & 0.60 & 1.13 & 0.87 \\
\hline \multicolumn{2}{|c|}{ Available Phosphorus } & 10.51 & 7.56 & 7.16 & 4.34 & 3.90 \\
\hline \multicolumn{2}{|l|}{ Base Saturation } & 79.08 & 74.05 & 82.98 & 86.55 & 7.26 \\
\hline \multicolumn{2}{|c|}{ Electrical Conductivity } & 0.010 & 0.010 & 0.0125 & 0.010 & 0.004 \\
\hline \multirow{5}{*}{ Exchangeable } & Calcium & 5.60 & 4.93 & 5.20 & 7.86 & 3.35 \\
\hline & Magnesium & 3.60 & 2.94 & 3.20 & 5.34 & 2.86 \\
\hline & Potassium & 0.083 & 0.053 & 0.063 & 0.065 & 0.018 \\
\hline & Sodium & 0.05 & 0.04 & 0.04 & 0.05 & 0.004 \\
\hline & Acidity & 2.48 & 2.76 & 1.72 & 1.92 & 1.10 \\
\hline \multicolumn{2}{|c|}{ Effective Cation Exchange Capacity } & 11.81 & 10.71 & 10.22 & 15.24 & 6.70 \\
\hline \multicolumn{7}{|c|}{30 - 60 cm Depth } \\
\hline \multicolumn{2}{|l|}{$\mathrm{pH}\left(\mathrm{H}_{2} \mathrm{O}\right)$} & 5.77 & 5.72 & 5.73 & 5.70 & 0.16 \\
\hline \multicolumn{2}{|c|}{ Organic Carbon } & 1.05 & 0.80 & 0.85 & 0.95 & 1.19 \\
\hline \multicolumn{2}{|c|}{ Available Phosphorus } & 11.00 & 10.69 & 9.11 & 9.34 & 4.92 \\
\hline \multicolumn{2}{|c|}{ Base Saturation } & 74.18 & 80.95 & 86.30 & 87.70 & 9.07 \\
\hline \multicolumn{2}{|c|}{ Electrical Conductivity } & 0.010 & 0.0175 & 0.020 & 0.0150 & 0.01 \\
\hline \multirow{5}{*}{ Exchangeable } & Calcium & 4.93 & 5.28 & 5.06 & 6.80 & 1.96 \\
\hline & Magnesium & 2.80 & 3.07 & 2.94 & 4.14 & 1.70 \\
\hline & Potassium & 0.065 & 0.048 & 0.057 & 0.050 & 0.030 \\
\hline & Sodium & 0.05 & 0.04 & 0.04 & 0.05 & 0.004 \\
\hline & Acidity & 2.69 & 1.92 & 1.34 & 1.72 & 0.93 \\
\hline \multicolumn{2}{|c|}{ Effective Cation Exchange Capacity } & 10.54 & 10.27 & 9.43 & 12.76 & 3.88 \\
\hline
\end{tabular}


Table 4. Ratios of some exchangeable bases.

\begin{tabular}{|c|c|c|c|c|c|}
\hline & \multicolumn{4}{|c|}{ Age of Oil Palm Plantation (Years) } & \multirow[b]{2}{*}{$\operatorname{LSD}_{0.05}$} \\
\hline & 0 & 9 & 29 & 49 & \\
\hline \multicolumn{6}{|l|}{0 - 15 cm Depth } \\
\hline Calcium/Magnesium & 1.59 & 1.62 & 1.69 & 1.73 & 0.59 \\
\hline Calcium/Potassium & 75.49 & 96.75 & 77.56 & 177.56 & 169.45 \\
\hline$($ Calcium + Magnesium $) /$ Potassium & 125.50 & 152.4 & 126.2 & 341.3 & 281.3 \\
\hline Magnesium + Potassium & 48.14 & 62.69 & 46.13 & 108.93 & 97.63 \\
\hline \multicolumn{6}{|l|}{15 - 30 cm Depth } \\
\hline Calcium/Magnesium & 1.64 & 1.70 & 1.64 & 1.51 & 0.30 \\
\hline Calcium/Potassium & 67.35 & 94.60 & 86.41 & 129.80 & 77.57 \\
\hline$($ Calcium + Magnesium $) /$ Potassium & 101.48 & 151.08 & 140.01 & 219.0 & 139.86 \\
\hline Magnesium + Potassium & 43.12 & 56.48 & 53.60 & 89.22 & 62.51 \\
\hline \multicolumn{6}{|l|}{30 - 60 cm Depth } \\
\hline Calcium/Magnesium & 1.77 & 1.74 & 1.74 & 1.74 & 0.33 \\
\hline Calcium/Potassium & 75.82 & 111.35 & 88.00 & 231.85 & 192.24 \\
\hline$($ Calcium + Magnesium $) /$ Potassium & 118.70 & 176.10 & 139.00 & 365.90 & 296.99 \\
\hline Magnesium + Potassium & 42.90 & 64.74 & 51.02 & 134.04 & 105.33 \\
\hline
\end{tabular}

Table 5. Extractable micronutrient contents $\left(\mathrm{mg} \cdot \mathrm{kg}^{-1}\right)$ of soils of the palm plantation.

\begin{tabular}{|c|c|c|c|c|c|}
\hline & \multicolumn{5}{|c|}{ Age of Oil Palm Plantation (Years) } \\
\hline & 0 & 9 & 29 & 49 & $\mathrm{LSD}_{0.05}$ \\
\hline \multicolumn{6}{|c|}{0 - 15 cm Depth } \\
\hline Manganese & 14.97 & 13.10 & 14.35 & 9.21 & 9.57 \\
\hline Zinc & 12.42 & 8.49 & 14.52 & 7.26 & 8.44 \\
\hline Copper & 9.29 & 9.54 & 13.07 & 15.17 & 6.71 \\
\hline Iron & 94.34 & 99.40 & 111.32 & 107.16 & 24.40 \\
\hline \multicolumn{6}{|c|}{15 - 30 cm Depth } \\
\hline Manganese & 13.25 & 11.41 & 12.00 & 4.60 & 5.27 \\
\hline Zinc & 23.99 & 9.65 & 8.30 & 7.84 & 10.08 \\
\hline Copper & 8.76 & 10.29 & 12.32 & 15.84 & 3.48 \\
\hline Iron & 96.89 & 96.31 & 107.67 & 104.75 & 24.41 \\
\hline \multicolumn{6}{|c|}{30 - $60 \mathrm{~cm}$ Depth } \\
\hline Manganese & 8.65 & 7.51 & 7.54 & 3.47 & 5.03 \\
\hline Zinc & 21.27 & 9.43 & 6.46 & 8.48 & 11.36 \\
\hline Copper & 9.35 & 9.75 & 11.49 & 15.39 & 4.73 \\
\hline Iron & 92.77 & 86.65 & 104.09 & 110.20 & 22.43 \\
\hline
\end{tabular}


Table 6. Regression equations for total weight (kg) of harvested oil palm bunches and corresponding quantity of oil (litres) produced versus environmental characteristics

\begin{tabular}{|c|c|}
\hline Dependent & Independent \\
\hline \multicolumn{2}{|c|}{ Micronutrients, Rainfall and Particle Size Fractions (PSF) } \\
\hline Total Weight of Bunches & $\begin{array}{l}=1308.163+0.075 \mathrm{Mn}(1)-5.796 \mathrm{Zn}(2)+48.751 \mathrm{Cu}(2)-19.826 \mathrm{Mn}(3)-9.408 \mathrm{Fe}(3)-0.590 \text { Coarse sand }(1) \\
\left(\mathrm{R}^{2}=0.98, \mathrm{p}<0.05\right)\end{array}$ \\
\hline Quantity of Palm Oil & $\begin{array}{l}=3230.275-0.033 \text { Rainfall }-125.551 \mathrm{Mn}(1)-34.031 \mathrm{Fe}(1)+0.512 \mathrm{Mn}(2)+380.597 \mathrm{Cu}(2)-54.397 \mathrm{Mn}(3)- \\
43.801 \mathrm{Fe}(3)+0.371 \text { Fine sand }(1)-2.736 \text { Coarse sand }(1)+5.180 \mathrm{Clay}(2)+0.162 \text { Coarse sand }(2)+4.257 \mathrm{Fine} \\
\text { sand }(3)+1.142 \text { Coarse sand }(3) \\
\left(\mathrm{R}^{2}=0.99, \mathrm{p}<0.05\right)\end{array}$ \\
\hline \multicolumn{2}{|c|}{ Rainfall and Other Soil Properties } \\
\hline Total Weight of Bunches & $\begin{array}{l}=-1067.015-0.053 \text { Rainfall }-5.520 \text { Available phosphorus }(1)+10.913 \text { Available phosphorus }(2)+ \\
\text { 4.805Available phosphorus }(3)-4.730 \text { Base saturation }(1)-1.562 \text { Base saturation }(2)-2.345 \text { Base saturation }(3) \\
+14.584 \text { Exchangeable acidity }(1)-779.269 \text { Electrial conductivity }(1)+13.597 \text { Effective cation exchange } \\
\text { capacity }(3)+25732 \text { Sodium }(2)+40.976 \text { Organic carbon }(1)+84.599 \text { Organic carbon }(2)+0.601 \mathrm{pH}(3) \\
\left(\mathrm{R}^{2}=0.99, \mathrm{p}<0.05\right)\end{array}$ \\
\hline Quantity of Palm Oil & $\begin{array}{l}=14865+0.280 \text { Rainfall }-249.682 \text { Available phosphorus }(1)-44.194 \text { Available phosphorus }(2)+4.221 \text { Base } \\
\text { saturation }(2)+42.750 \text { Exchangeable acidity }(2)-53923 \text { Electrical conductivity }(1)-21758 \text { Electrical } \\
\text { conductivity }(3)-41.701 \text { Effective cation exchange capacity }(1)+9.792 \text { Effective cation exchange } \\
\text { capacity }(2)+49.374 \text { Effective cation exchange capacity }(3)+107905 \text { Sodium }(1)-287.107 \text { Organic carbon }(2) \\
-2999.668 \mathrm{pH}(1)-15.773 \mathrm{pH}(3) \\
\left(\mathrm{R}^{2}=0.99, \mathrm{p}<0.05\right)\end{array}$ \\
\hline
\end{tabular}

$1,2,3$ in parenthesis corresponds to $0-15 \mathrm{~cm}, 15-30 \mathrm{~cm}$ and $30-60 \mathrm{~cm}$ depths respectively.

Table 7. Absolute contribution of soil properties and rainfall to the modeling of palm oil production in the study area ${ }^{+}$

\begin{tabular}{|c|c|c|c|c|c|}
\hline \multirow{2}{*}{ Independent } & \multicolumn{2}{|c|}{ Dependent (\%) } & \multirow{2}{*}{ Independent } & \multicolumn{2}{|c|}{ Dependent (\%) } \\
\hline & $\mathrm{FFB}^{*}$ & Palm Oil & & Palm Oil & $\mathrm{FFB}^{*}$ \\
\hline Micronutrients & & & Other Soil Properties & & \\
\hline Intercept & 39.88 & 18.15 & Intercept & 14.61 & 38.16 \\
\hline Rainfall & - & 1.46 & Rainfall & 5.71 & 5.65 \\
\hline 0 - 15 cm Depth & & & 0 - 15 cm Depth & & \\
\hline Manganese & 0.03 & 9.10 & $\mathrm{pH}(\mathrm{H} 2 \mathrm{O})$ & - & 44.90 \\
\hline Iron & - & 19.70 & Organic Carbon & 0.71 & 0.00 \\
\hline 15 - 30 cm Depth & & & Available Phosphorus & 0.35 & 2.93 \\
\hline Manganese & - & 0.03 & Base Saturation & 5.25 & 0.00 \\
\hline Zinc & 2.20 & - & Electrical Conductivity & 0.15 & 1.99 \\
\hline Copper & 17.54 & 25.23 & Exchangeable Sodium & 66.50 & 0.00 \\
\hline 30 - 60 cm Depth & & & Effective Cation Exchange Capacity & - & 1.17 \\
\hline Manganese & 4.11 & 2.08 & 15 - 30 cm Depth & & \\
\hline Iron & 28.23 & - & Organic Carbon & 1.14 & 0.72 \\
\hline 0 - 15 cm Depth & & & Available Phosphorus & 0.49 & 0.84 \\
\hline Coarse Sand & 8.00 & 6.84 & Base Saturation & 1.73 & 0.87 \\
\hline Fine Sand & - & 0.81 & Exchangeable Acidity & 0.03 & 0.00 \\
\hline 15 - 30 cm Depth & & & Effective Cation Exchange Capacity & - & 0.30 \\
\hline Coarse Sand & - & 0.36 & 30 - 60 cm Depth & & \\
\hline Clay & - & 3.11 & $\mathrm{pH}\left(\mathrm{H}_{2} \mathrm{O}\right)$ & 0.05 & 0.23 \\
\hline 30 - 60 cm Depth & & & Available Phosphorus & 0.66 & 0.00 \\
\hline Coarse Sand & & 2.47 & Base Saturation & 2.64 & - \\
\hline Fine Sand & & 10.65 & Electrical Conductivity & - & 0.87 \\
\hline & & 100 & Effective Cation Exchange Capacity & - & 1.36 \\
\hline
\end{tabular}

${ }^{+}$variable that occurred in the table are those that influence the modelling process; ${ }^{*}$ Fresh Fruit Bunch. 
affected their distribution.

The contribution of micronutrients in the growth and development of oil palm may be responsible for the significant $(\mathrm{p}<0.05)$ differences in manganese $(\mathrm{Mn})$, zinc $(\mathrm{Zn})$ and copper $(\mathrm{Cu})$ at $15-60 \mathrm{~cm}$ depth, and $\mathrm{Fe}$ at 30 $60 \mathrm{~cm}$ (Table 5). The micronutrients at the surface $(0$ $15 \mathrm{~cm}$ depth) soil were not significantly different at the various age groups of the oil palm plantation. Whereas soil on the $15-30 \mathrm{~cm}$ depth indicated that manganese content at the control, 9 years and 29 years old oil palm plantations were not significantly different from each other. A more impressive result was found in zinc where the effect of land use resulted in locations with oil palm plantation not significantly different from each other (ranging between $9.65 \mathrm{mg} \cdot \mathrm{kg}^{-1}$ and $7.84 \mathrm{mg} \cdot \mathrm{kg}^{-1}$ ) but significantly different from the control $\left(23.99 \mathrm{mg} \cdot \mathrm{kg}^{-1}\right)$. This was a confirmation that zinc is required for proper growth and development of oil palm. Additionally, the effect of large biomass of oil palm that tend to sequester large amount of soil nutrients and additional removals in FFB contribute to locations with oil palm manifesting lower nutrient status [5]. The reverse of the $\mathrm{Zn}$ trend was observed in $\mathrm{Cu}$ content at $15-30 \mathrm{~cm}$ depth with the control and 9 years old oil palm plantation not significantly different from each other. This may be an indication that as oil palm gets older, it tends to release some of extractable copper previously sequestered in the dry matter back to the soil. The trend of distribution of $\mathrm{Mn}, \mathrm{Zn}$ and $\mathrm{Cu}$ at the $15-30 \mathrm{~cm}$ depth was replicated at the $30-60$ $\mathrm{cm}$ depth. The only exception was in the distribution of extractable iron which had not been found to have significantly changed at other depths but $30-60 \mathrm{~cm}$ depth. These typically indicated that different nutrients are either required or taken up at different depths within the 0 $60 \mathrm{~cm}$ depths reported as effective feeding depth for oil palm roots [2].

Multiple stepwise regression analysis (Table 6) was used to separately model the relationship between the soil properties measured at various depths and either fresh fruit bunch (FFB) yield $\left(\mathrm{kg} \cdot \mathrm{ha}^{-1} \cdot \mathrm{yr}^{-1}\right)$ or the quantity of palm oil generated therein (litres $\mathrm{ha}^{-1} \cdot \mathrm{yr}^{-1}$ ). The analyses were carried out in two stages for each yield parameter (i.e. FFB and palm oil) and the results were as shown in Table 6. The first stage modeled micronutrients, rainfall and particle size fractions (PSF) against either FFB or palm oil, while the second stage modeled other soil properties and rainfall. The discrimination was made between micronutrients and other soil properties as a result of the perceived importance of micronutrients in oil palm production and to reduce the quantity of data that will be used in modeling to a manageable size. This is not without mindfulness of the fact that their availability may influence each other. Therefore the soil proper- ties that significantly contribute to the models were brought together as the dimensionality had been drasticcally reduced. But it was impossible to establish in the models that the variable could act together. Yet the integration of rainfall and PSF in the various stages of the modeling process was in consideration of the importance of PSF in solute transport [29] and that of water from the rainfall especially in coastal plain sands soils [30-32].

Generally, rainfall was identified as an integral as it manifested its importance in almost the entire models. The contribution of rainfall manifested more in the other soil properties and to a less extent in micronutrients. It was observed that the relationship between micronutrients and FFB largely depended on the manganese content at the $0-15 \mathrm{~cm}$ and $30-60 \mathrm{~cm}$ depth, zinc and copper at the $15-30 \mathrm{~cm}$ depth and coarse sand at the $0-15 \mathrm{~cm}$ depth. The micronutrients present in the modeling of quantity of palm oil included $\mathrm{Mn}$ (0 - $60 \mathrm{~cm}$ depth), $\mathrm{Fe}(0$ $-15 \mathrm{~cm}$ and $30-60 \mathrm{~cm}$ depths), $\mathrm{Cu}(15-30 \mathrm{~cm}$ depth) and particle size fractions. In consideration of other soil properties, the importance of available phosphorus and base saturation especially in the FFB and ECEC in the quantity of palm oil was manifested in the modeling process. In as much as the exchangeable bases were not found to have significantly influenced production, the participation of ECEC indicated that compound fertility indicators are more important than their individual contribution. The presence of negative signs in the models indicated that in-as-much-as those nutrients were required, increases beyond a particular threshold that was not determined in this study may be detrimental to the performance of the crop.

The relative contribution of the different variables to the model equation was computed as the mean values of the variables (Table 1) multiplied by their coefficients in the regression equation or model and expressed as the percentage of the absolute total (i.e. irrespective of accompanying signs), their values will determine corresponding contribution they made in the overall value of the dependent variables. This means that the variable which posses highest value will exert highest influence either in increasing or decreasing yield. Table 7 indicated that effects of PSF are minimal while exchangeable sodium was highest in overall contribution (i.e. 66.5 percent) in the quantity of oil palm production. Also extractable copper and zinc were found to have made large contributions to FFB and oil palm. The unexplained (intercept) was very similar and high in the FFB for the soil properties indicative that the outcome of the modeling activity may not be sporadic as the overall level of significance was $<5 \%$ and the model $\mathrm{R}^{2}$ for the processes was $>0.98$. 


\section{Conclusion}

The oil palm may have influenced soil development with its effect on the silt content at the $30-60 \mathrm{~cm}$ depth. The soil properties apart from micronutrients and texture that vary significantly among the land uses are equally those that significantly influence the modeling process. Land use was found to significantly influence the micronutrients in the soils. The importance of zinc and its removal resulted in significantly higher zinc contents in control compared to oil palm bearing soils. Sequestration of nutrients in large biomass and removals in FFB of oil palm grossly diminishes soil nutrients and creates the need for proper nutrient management in oil palm enterprises. Notwithstanding pragmatic complexities that may be involved, if locations (i.e. depth) and yield components were adequately considered in the planning and management of nutrient supplementation, there may be increases and minimal variability in the yield of fresh fruit bunch and palm oil especially on coastal plain sands soils.

\section{REFERENCES}

[1] M. Rieger, "Introduction to Fruit Crops," Haworth Press, Inc., New York, 2006.

[2] E. Mutert, "Suitability of Soils for Oil Palm in Southeast Asia," Better Crops International, Vol. 13, No. 1, 1999, pp. 36-38.

[3] A. E. Imogie, C. V. Udosen and M. M. Ugbah, "Fertility Indices and Management of Hydromorphic Soils Supporting Raphia Palm (Raphia hookeri) Mann and Wend Land) Plantation at Onuebum, Bayelsa State, Nigeria," Continental Journal of Agronomy, Vol. 2, 2008, pp. 1924.

[4] K. O. Soyebo, A. J. Farinde and E. D. Dionco-Adetayo, "Constraints of Oil Palm Production in Ife Central Local Government Area of Osun State," Nigerian Journal of Soil Science, Vol. 10, No. 1, 2005, pp. 55-59.

[5] A. M. Tarmizi, "Nutritional Requirements and Efficiency of Fertilizer Use in Malaysian Oil Palm Cultivation," In: B. Yusof, B. S. Jalani and K. W. Chan, Eds., Advances in Oil Palm Research, 2000, pp. 411-440.

[6] S. K. Ng, "Review of Oil Palm Nutrient and Manuring: Scope for Greater Economy in Fertilizer Usage," Oleagineux, Vol. 32, No. 5, 1977, pp. 197-209.

[7] S. K. Ng, Y. P. Tan, E. Chan and S. P. Cheong, "Nutritional Complexes of Oil Palms Planted on Peat Soil in Malaysia. II. Preliminary Results of Copper Sulphate Treatments," Oleagineux, Vol. 29, No. 10, 1974, pp. 445456.

[8] G. Singh, "Micronutrient Studies of the Oil Palm on Peat," The Seminar on Fertilizers in Malaysian Agriculture, Serdang, 28 March 1983, pp. 763-779.

[9] SLUS-AK, "Soils and Land Use Survey of Akwa Ibom," Government Printers, Uyo, 1989.
[10] A. G. Ojanuga, G. Lekwa and F. O. R. Akamigbo, "Survey, Classification and Genesis of Acid Sands," In: E. J. Udo and R. A. Sobulo, Eds., Acid Sands of Southeastern Nigeria, Soil Science Society of Nigerian, 1981, pp. 1-7.

[11] G. E. K. Ofomata, "Actual and Potential Erosion in Nigeria and Measures for Control," In: E. J. Udo and R. A. Sobulo, Eds., Acid Sands of Southeastern Nigeria, Soil Science Society of Nigerian, 1981, pp. 151-165.

[12] G. Lekwa and E. P. Whiteside, "Coastal Plain Soils of Southeastern Nigeria: I. Morphology, Classification and Genetic Relationship," Soil Science Society of America Journal, Vol. 50, No. 1, 1986, pp. 154-160.

[13] G. W. Gee and J. W. Bauder, "Particle Size Analysis," In: A. Klute, Ed., Methods of Soil Analysis: Part 1, Agronomy Society of America and Soil Science Society of America, Madison, 1986. pp. 404-407.

[14] D. W. Nelson and I. E. Sommer, "Total Carbon, Organic Carbon and Organic Matter," In: A. I. Page, Ed., Methods of Soil Analysis, Part II, Agronomy Society of America and Soil Science Society of America, Madison, 1982, pp. 961-1010.

[15] E. O. McLean, "Soil pH and Lime Requirement," In: A. I. Page, Ed., Methods of Soil Analysis, Part II, Agronomy Society of America and Soil Science Society of America, Madison, 1982, pp. 199-223.

[16] A. Mehlick, "Mehlick 3 Soil Test Extractant: A Modification of Mehlick 2 Extractant," Communications in Soil Science and Plant Analysis, Vol. 15, No. 12, 1984, pp. 1409-1416. doi:10.1080/00103628409367568

[17] J. M. Anderson and J. S. I. Ingram, "Tropical Soil Biology and Fertility. A Handbook of Methods," 2nd Edition, CAB International, Wallingford, 1993.

[18] Soil Survey Staff, "Keys to Soil Taxonomy," United States Department of Agriculture," Natural Resources Conservation Services, Washington DC, 2006.

[19] S. S. Shapiro and M. B. Wilk, "An Analysis of Variance Test for Normality,” Biometrika, Vol. 52, No. 3-4, 1965, pp. 691-710.

[20] SAS Institute, "SAS/STST User's Guide, Version 6," 4th Edition, SAS Institute, Inc., Cary, 1989.

[21] L. P. Wilding, "Spatial Variability: Its Documentation, Accommodation, and Implication to Soil Surveys," In: D. R. Nielsen and J. Bouma, Eds., Soil Spatial Variability, Pudoc., Wageningen, 1985, pp. 166-194.

[22] T. O. Ibia, G. S. Effiong, P. I. Ogban and J. C. Obi, "Relationship between Phosphorus Forms and Parent Materials in Soils of Southeastern Nigeria," Acta Agronomica Nigeriana, Vol. 8, No. 2, 2009, pp. 104-112.

[23] J. C. Obi and A. O. Ogunkunle, "Influence of Termite Infestation on the Spatial Variability of Soil Properties in the Guinea Savanna Region of Nigeria," Geoderma, Vol. 148, No. 3-4, 2009, pp. 357-368.

[24] A. O. Olaleye, A. O. Ogunkunle and K. L. Sahrawal, "Forms and Pedogenic Distribution of Extractable Iron in Selected Wetland Soils in Nigeria," Communications in Soil Science and Plant Analysis, Vol. 31, No. 7-8, 2000, pp. 923-941. doi:10.1080/00103620009370488 
[25] J. D. Phillips, "Soil System Modelling and Generation of Field Hypotheses," Geroderma, Vol. 145, No. 3-4, 2008, pp. 419-425. doi:10.1016/i.geoderma.2007.07.001

[26] J. D. Shaw, E. C. Sr. Packee and C. L. Ping, "Growth of Balsam Poplar and Black Cottonwood in Alaska in Relation to Landform and Soil," Canada Journal of Soil Resources, Vol. 31, No. 10, 2001, pp. 1793-1804.

[27] R. L. Voortman, J. Brouwer and P. J. Albersen, "Characterization of Spatial Soil Variability and Its Effect on Millet Yield on Sudano-Sahelian Coversands in SW Niger," Geoderma, Vol. 121, No. 1-2, 2002, pp. 65-82.

[28] J. C. Obi, G. E. Akinbola, A. O. Ogunkunle and A. O. Umeojiakor, "Profile Distribution of Clay, $\mathrm{Ca}, \mathrm{Mg}$ and $\mathrm{K}$ in Some Soils of the Savanna Region of Nigeria," Journal of Tropical Agriculture, Food, Environment and Extension, Vol. 9, No. 2, 2010, pp. 76-83.

[29] J. S. Strock, D. K. Cassel and M. L. Gumpertz, "Spatial
Variability of Water and Bromide Transport through Variably Saturated Soil Blocks," Soil Science Society of America Journal, Vol. 65, No. 6, 2001, pp. 1607-1617. doi:10.2136/sssaj2001.1607

[30] M. Duffera, J. G. White and R. Weisz, "Spatial Variability of Southeastern U. S. Coastal Plain Soil Physical Properties: Implication for Site-Specific Management," Geoderma, Vol. 137, No. 3-4, 2007, pp. 327-339.

[31] T. O. Ibia, I. B. Uko-Haka, S. O. Edem, P. I. Ogban and J. C. Obi, "Evaluation of the Acid Soils for Sanitary Landfills in Akwa Ibom State, Southeastern Nigeria," Nigerian Journal of Soil Science, Vol. 21, No. 1, 2011, pp. 1-5.

[32] P. I. Ogban and I. O. Ekerette, "Physical and Chemical Properties of the Coastal Plain Sands Soils of Southeastern Nigeria," Nigeria Journal of Soil Research, Vol. 2, 2001, pp. 6-14. 